\title{
THE CONSTRUCTION OF HOMOGENEOUS CONVEX CONES
}

\author{
BY OSCAR S. ROTHAUS \\ Communicated by G. A. Hedlund, November 19, 1962
}

An open convex cone $D$ in $E^{n}$ is called homogeneous if the subgroup of linear transformations of $E^{n}$ leaving $D$ invariant, called the group of automorphisms of $D$, acts transitively on $D$. In this note we indicate briefly an inductive procedure by means of which all homogeneous convex cones may be constructed. Details of the construction will be given elsewhere.

The dual of the convex cone $D$ is defined to be the set

$$
D^{\prime}=\text { interior }\left\{x \in E^{n} \mid x t^{t} \geqq 0 \text { for all } y \in D\right\} .
$$

$D^{\prime}$ is also an open convex cone, and $D$ is called regular if $D^{\prime}$ is nonempty. Every open convex cone is "equivalent" to the Cartesian product of a Euclidean space and a regular cone. (Two cones $D_{1}$ and $D_{2}$ in $E^{n}$ are called equivalent if there is a nonsingular linear mapping of $E^{n}$ throwing $D_{1}$ onto $D_{2}$.) It obviously suffices to construct all homogeneous regular cones. Aside from their intrinsic interest, the importance of regular cones lies in the fact that the "complex tube" [4] over such a cone is analytically equivalent to a bounded homogeneous analytic manifold. Furthermore, it may be shown that the corresponding analytic manifold is symmetric (in the sense of $\mathrm{E}$. Cartan) if and only if the cone is self-adjoint, i.e., equivalent to a cone equal to its own dual. Self-adjoint cones have been studied by several authors [1-5], and completely classified. The self-adjoint cones may also be characterized as having a completely reducible group of automorphisms.

The connection of this topic with the question of "domains of positivity" studied earlier by Koecher [1-3] and the author [4] lies in the following simple observation. Let $D$ be a regular cone and $D^{\prime}$ its dual. Then the set of pairs $(x, y), x \in D, y \in D^{\prime}$, is a domain of positivity with characteristic the symmetric matrix

$$
\left[\begin{array}{ll}
0 & I \\
I & 0
\end{array}\right]
$$

where $I$ is the identity matrix of appropriate dimension. Since $D^{\prime}$ is homogeneous if $D$ is, the study of homogeneous regular cones is more or less the same as the study of homogeneous domains of positivity. 
Before going on to the construction, we wish to emphasize that our procedure is not strictly a classification, since in the inductive construction, a given cone may arise more than once. Vinberg $[6 ; 7]$ has indicated a 1-1 correspondence between regular cones and a certain class of nonassociative algebras, but the construction of the algebras seems somewhat obscure. Furthermore, his correspondence does not seem to me to bring out the natural geometric manner by which such cones arise.

A well-known class of homogeneous regular cones is for each $n$ the set $S^{n}$ of $n \times n$ positive definite symmetric matrices. Then the set of points $(x, y, z), x \in E^{1}, y \in E^{n}, z \in S^{n}$, such that $x>y z^{-1} y^{t}$ is evidently the cone $S^{n+1}$. This simple inductive procedure for constructing $S^{n+1}$ given $S^{n}$ supplies the model for the construction of all homogeneous regular cones. To be more precise, we must make some preliminary remarks.

Let $D$ be a homogeneous regular cone in $E^{n}$. By a "representation" of degree $r$ of $D$ we mean a linear mapping $z \rightarrow U(z)$ of $E^{n}$ into the space of $r \times r$ symmetric matrices such that:

(1) $U(z)$ is positive definite for $z \in D$.

(2) Corresponding to each element $H$ of some transitive subgroup of the group of automorphisms of $D$ there is an $r \times r$ matrix $\phi(H)$ such that we have identically in $z: \phi(H) U(z H) \phi(H)^{t}=U(z)$.

Given such a representation $U(z)$ of $D$, it is readily verified that the set $P$ of points $(x, y, z), x \in E^{1}, y \in E^{r}, z \in D$, such that $x>y U^{-1}(z) y^{t}$ is a regular cone. We call $P$ an extension of $D$ by the representation $U$. Furthermore, $P$ is homogeneous, and a transitive group of automorphisms of $P$ is generated by the following three types of linear transformation:

(1) $(x, y, z) \rightarrow\left(x+y b^{t}+\frac{1}{4} b U(z) b^{t}, y+\frac{1}{2} b U(z), z\right)$ for all $b \in E^{\text {r. }}$

(2) $(x, y, z) \rightarrow(x, y \phi(H), z H)$.

(3) $(x, y, z) \rightarrow\left(\alpha x, \alpha^{1 / 2} y, z\right)$ for all $\alpha>0$.

We have now the following result:

THEOREM 1. Starting with the half-line $z>0$, the above described process of extension, repeated over and over, yields all homogeneous regular cones.

The proof of this result depends upon a detailed study of the group of automorphisms of a given cone. In connection with this study, we found the following statement quite useful.

THEOREM 2. Let $G$ be a closed transitive subgroup of the group of automorphisms of a regular cone $D$ and let $H$ be a closed connected normal subgroup of $G$ such that $G / H$ is compact. Then $H$ is transitive on $D$. 
On the basis of Theorem 1, in order to construct all homogeneous cones it suffices to construct all representations of a given one. (We note in passing that every representation of a cone is in an obvious manner a direct sum of irreducible representations.) This program is also carried out inductively. Namely, let $P$ be a homogeneous regular cone. We wish to construct all representations of $P$. By Theorem 1, $P$ consists of points $(x, y, z) x \in E^{1}, y \in E^{r}, z \in D$, such that $x>y U^{-1}(z) y^{t}$, where $D$ is some regular cone and $U$ some representation of $D$. The possible representations of $P$ are now deduced from those of $D$. By appropriate choice of coordinate system in $E^{*}$, a given representation $V=V(x, y, z)$ of $P$ of degree $s$ may be brought into the following form:

$$
V(x, y, z)=\left[\begin{array}{cc}
x I & B(y) \\
B(y)^{t} & R(z)
\end{array}\right]
$$

where $R(z)$ is some representation of $D, I$ is an identity matrix, and the matrix $B(y)$ satisfies the equation $B(y) B(y)^{t}=\left(y y^{t}\right) I$. This together with certain group invariance properties of $B(y)$, depending on the representation $R(z)$, enable us to enumerate all possibilities for $B(y)$. The complete statement requires too much detail for us to go into here. It is interesting to note, however, that the construction of $B(y)$ depends in some cases on representations of Clifford algebras, this being the mechanism by which Cayley numbers enter the picture.

\section{REFERENCES}

1. M. Koecher, Positivitatsbereiche im $R^{n}$, Amer. J. Math. 79 (1957), 575-596.

2. - Analysis in reelen Jordan-Algebren, Nachr. Akad. Wiss. Göttingen Math.-Phys. Kl. II 1958, 67-74. 202.

3. - Die Geodätischen von positivitätsbereichen, Math. Ann. 135 (1958), 192-

4. O. Rothaus, Domains of positivity, Abh. Math. Sem. Univ. Hamburg 24 (1960), 189-235.

5. E. B. Vinberg, Homogeneous cones, Dokl. Akad. Nauk SSSR 133 (1960), 9-12. (Russian)

6. - Convex homogeneous domains, Dokl. Akad. Nauk SSSR 141 (1961), 521-524. (Russian)

7. - Automorphisms of homogeneous convex cones, Dokl. Akad. Nauk. SSSR 143 (1962), 265-268. (Russian)

Institute for Defense ANALyses 